\title{
A Survey of the Incidence of Neonatal Sepsis by Group B Streptococcus During a Decade in a Brazilian Maternity Hospital
}

Edinéia Vaciloto, Rosana Richtmann

Helenilce de Paula Fiod Costa, Elisa Junko Ura Kusano,

Santa Joana Hospital

Maria Fernanda Branco de Almeida and Eduardo Rahme Amaro

\begin{abstract}
Group B Streptococcus (GBS) is the main etiological agent of neonatal sepsis in developed countries, however there is no detailed information on its incidence in Brazil. We registered the incidence and lethality of GBS infection in a Brazilian private maternity hospital from April 1991 to March 2000. Maternal risk factors contributing to neonatal infections were also scored. The rate of infection was determined by checking for GBS in the blood and liquor of symptomatic neonates within 72 hours of birth. Sepsis and/or early onset meningitis were diagnosed in 43 neonates (32 cases in blood, 1 in liquor and 10 in blood and liquor). The overall incidence was 0.39 per thousand neonates and remained quite constant throughout the period, ranging from 0.25 0.63. Septic shock occurred in 33 neonates within 1 to 36 hours of birth (mean 15 hours). Among those patients, $26(60 \%)$ died between the $5^{\text {th }}$ and the $85^{\text {th }}$ hour after birth. Maternal risk factors, according to CDC criteria, included: gestational age below 37 weeks in 26 cases $(60 \%)$, amniorrhexis equal or superior to 18 hours in 7 cases $(16 \%)$, and maternal temperature equal or superior to $38^{\circ} \mathrm{C}$ in 4 cases $(9 \%)$. None of the mothers had received prophylactic antibiotics during labor nor were urine, rectal or vaginal swabs screened for GBS. Although the incidence of GBS infection in the population in this study was lower than that found in developed countries, its rate of mortality was higher. The death rate could be reduced through recognition of the risk factors and prophylactic antibiotics during labor.
\end{abstract}

Key Words: Sepsis, newborn, Group B Streptococcus.

During the last three decades, group B Streptococcus (GBS) has been the most frequent etiological agent of neonatal sepsis in developed countries, being responsible for high morbidity-mortality rates. GBS is also an important agent of maternal infections, such as chorioamnionitis, endometritis, urinary and surgical-site infections [1,2]. The epidemiology of this disease, both in pregnant women and neonates, has been extensively studied in the United States and Europe but not so in Latin America. Group

Received on 28 June 2001; revised 26 March 2002.

Address for correspondence: Dr. Edinéia Vaciloto. Rua Paraíso, 432. Phone: (11) 5080-6068. Fax: (11) 5539-7460

E-mail: centrodeestudos@hmsj.com.br

The Brazilian Journal of Infectious Diseases 2002;6(2):55-62 (C) 2002 by The Brazilian Journal of Infectious Diseases and Contexto Publishing. All rights reserved.

$1413-8670$
B streptococcus is synonymous with Streptococcus agalactiae in the obstetric as well as in the pediatric literature and in this paper we maintain this meaning, which is in common use [1].

There are two forms of neonatal sepsis: one earlyonset or precocious, and the other late-onset. The former occurs during the first seven days of life, corresponding to $80 \%$ of all cases, and is due to vertical transmission during labor or birth. It includes bacteremia and/or sepsis, meningitis and pneumonia. Respiratory symptoms usually flare up during the first 24 hours after birth and are similar to those of hyaline membrane disease. Sepsis, which may evolve to septic shock, occurs in $25 \%$ to $40 \%$ of the neonates and meningitis in $5 \%$ to $15 \%$.

Late-onset sepsis affects neonates from one to seven weeks of age. Its transmission can be vertical, horizontal or nosocomial. Its most common clinical manifestations 
are: meningitis (30\% to $40 \%$ ), bacteremia (40\%), septic arthritis (5\% to $10 \%)$ and, more rarely, omphalitis and osteomyelitis.

The worldwide incidence of early-onset sepsis is around 0.7 to 3.7 cases per thousand neonates, whereas the incidence of late-onset sepsis is about 0.5 to 1.8 per thousand neonates. Up to the 1970's, lethality in developed countries was around $15 \%$ to $55 \%$, but during the last few years, it has dropped to $10 \%$ to $15 \%$ for the early-onset form and to $2 \%$ to $6 \%$ for late-onset sepsis [2-10]. There are no data about the lethality and mortality of neonatal sepsis in Brazil.

The determining factor diagnosis of neonatal sepsis is the presence of GBS in the mother's genital tract during birth. The distal third of the vaginal mucosa may be colonized by GBS from the anorectal canal. For that reason, diagnostic swabs are directed to these areas. The urinary tract is also an important site for infection, especially during pregnancy, when it manifests as asymptomatic bacteriuria $[1,3,11]$.

The prevalence of maternal colonization by GBS depends on the swab source (vagina or rectum), time of pregnancy, race, origin, age, parity and socialeconomic level. These variables can explain the wide variation of positive swabs (from 5\% to $41 \%$ ) in studies conducted in different countries. Prospective studies indicate that vertical transmission varies from $29 \%$ to $85 \%$, with an average of $51 \%[3,11-16]$.

The risk of neonates being infected by vertical transmission is directly related to the absolute number of microorganisms in the birth canal during delivery. The neonate born of a colonized mother is more prone to develop invasive disease; however, only $1 \%$ to $2 \%$ of babies born of women with a positive vaginal/rectal swab develop neonatal sepsis. This frequency increases if one or more maternal risk factors are present $[3,12,13]$. In a 1996 publication, the American Academy of Pediatrics (AAP), the American College of Obstetricians and Gynecologists (ACGO) and the Centers for Disease Control (CDC) informed that the most important factors included: previous history of fraternal infection by an invasive disease, bacteriuria caused by GBS during pregnancy, birth before the $37^{\text {th }}$ week of gestation, membrane rupture after 18 hours or more of labor, mother's temperature equal or superior to $38^{\circ} \mathrm{C}$, in addition to the presence of GBS in the vagina and/or rectum [15,17-19]. Other risk factors have also been reported, including low neonatal weight, multiparity and asphyxia at birth $[1,3,20]$.

Adequate maternal diagnosis and treatment are necessary to reduce the neonatal morbidity and mortality associated with this disease. Many studies conducted in the U.S. confirm that prophylactic ampicillin and/or intravenous penicillin during labor in women with the above-mentioned risk factors would significantly reduce the vertical transmission of GBS and consequent neonatal sepsis [13,16-22].

Based on these considerations, our primary objective was to determine the incidence and lethality of neonatal sepsis and/or meningitis caused by GBS in the 1990's in a private Brazilian maternity hospital, and also to describe maternal risk factors associated with these infections.

\section{Material and Methods}

An analysis was made of the records of all neonates affected with sepsis and/or meningitis who presented symptoms within 72 hours of birth. These patients were treated at the Intensive Care Unit of the Hospital e Maternidade Santa Joana from April 1991 to March 2000.This is a private institution situated in São Paulo, Brazil, which cares for about 14,000 neonates /year with a hospital stay of, at least 72 hours. The Hospital Ethics Committee approved the study.

The subjects were neonates who had sepsis and/ or meningitis and presented hyper or hypothermia, apnea, bradycardia and/or shock during the first 72 hours after birth, and had GBS in the blood and/or liquor [23].

Blood culture was performed manually: $0.5 \mathrm{ml}$ to $1.0 \mathrm{ml}$ of blood was inoculated into liquid broth (Soya Tryptone Broth), incubated at $35^{\circ} \mathrm{C}$ and then plated onto blood agar medium. Liquor was inoculated into chocolate agar medium, and in glucose and thioglycolate broth and incubated at $35^{\circ} \mathrm{C}$ in a $10 \%$ 
carbon dioxide atmosphere. Isolated bacteria colonies were identified through the traditional methods of catalase, CAMP and the latex test. The antibiotic resistance test was done according to the Kirby-Bauer method [24].

Lethality and mortality by GBS were scored on a yearly basis. The following maternal and neonate data were recorded: prenatal follow up, parity, gestational age, use of antibiotics, type of delivery, weight at birth, the weight/gestational age relation [25], sex, Apgar score at the first and fifth minute of life, medication taken at delivery and the clinical evolution of sepsis.

Maternal risk factors were evaluated according to AAP, ACGO and CDC protocols (1996). These factors included: previous history of neonatal sepsis in a sibling, GBS bacteriuria during pregnancy, gestational age under 37 weeks, membrane rupture equal or superior to 18 hours, maternal temperature equal or superior to $38^{\circ} \mathrm{C}$ and vaginal and/or anorectal presence of GBS during pregnancy and delivery.

A linear progression test was used to analyze the yearly incidence of neonatal sepsis.

\section{Results}

During the period of study, 43 out of 111,241 babies developed neonatal sepsis/meningitis within 72 hours of birth. Therefore, the incidence of invasive neonatal disease was 0.39 per thousand live births, with a minimum value of 0.25 and a maximum of 0.63 . Statistical analysis showed that the rate of neonatal GBS infection did not change over the years $(p=0.147)$. Among the 43 patients, 26 (60\%) died (Table 1).

Maternal and demographic data on 43 neonates are shown in Table 2 . In most cases (60\%), the mother was primipara. All of them had had pre-maternal care; the average gestational age was 35.7 weeks and the median 36.4 (minimum value 23 and maximum 40 weeks). Thirteen neonates had a gestational age of less than 35 weeks, 17 patients were 35 to 37 weeks and 13 between 38 and 40 weeks. None of the mothers received antibiotic treatment during labor; 28 (65\%) of the births were by cesarean section. The average weight at birth was $2,570 \mathrm{~g}$ and the median $2,640 \mathrm{~g}$ (minimum 550g and maximum 4,360g); 40 (93\%) had an adequate weight and $19(42 \%)$ were boys. In the first and fifth minutes after birth, respectively, 18 (42\%) and $5(12 \%)$ of the neonates had an Apgar score less than or equal to 7 . Two patients received vasoactive drugs immediately after birth (Table 2).

Among the 43 neonates, 32 (72\%) presented sepsis, with a positive blood culture for GBS, 10 (23\%) had sepsis associated with meningitis and GBS in the blood and liquor, whereas one neonate had meningitis and GBS solely in the liquor. Considering all cases, 33 (77\%) of the neonates eventually went into septic shock and had to undergo vasoactive therapy to maintain homeostasis. The symptoms of shock appeared an average of 15.5 hours after birth (1 to 36 hours) and death occurred a mean of 41 hours after birth (from 5 to $85 \mathrm{hrs})$ in 26 patients $(60 \%)$.

Maternal risk factors included: gestational age inferior to 37 weeks in 26 patients $(60 \%)$, membrane rupture superior to 18 hours in 7 cases $(16 \%)$ and maternal temperature superior to $38^{\circ} \mathrm{C}$ in 4 cases $(9 \%)$. Among the 17 non-primipara patients, 9 indicated previous miscarriages, 2 had had stillborn infants and one a neonatal death of unknown cause.

No clinical risk factors were detected in 14 patients (33\%). However, none of the patients had a record of bacteriuria by GBS during pregnancy. None of the mothers had a GBS vaginal and/or rectal swab test during pregnancy and/or labor. Risk factors are specified in Table 3.

\section{Discussion}

During the last 30 years, many published reports have confirmed that GBS is the most frequent cause of early neonatal infection in developed countries $[2,4,11,13,22,23,26-28]$. We determined the incidence of GBS infection in the 90's in a private maternity hospital where all patients undergo prenatal follow up. We studied the clinical outcome and laboratory profile of neonatal GBS infection, as well as the associated maternal risk factors detected 
Table 1. Incidence and lethality of meningitis/sepsis by Group B Streptococcus (GBS) in neonates at Hospital e Maternidade Santa Joana

\begin{tabular}{lcccc}
\hline & $\begin{array}{c}\text { Number of } \\
\text { neonates } \\
\text { infected by } \\
\text { GBS }\end{array}$ & $\begin{array}{c}\text { Total number } \\
\text { of liveborns }\end{array}$ & $\begin{array}{c}\text { Incidence of } \\
\text { GBS infection } \\
\text { per 1,000 } \\
\text { neonates }\end{array}$ & $\begin{array}{c}\text { Lethality (\%) } \\
\text { due to GBS } \\
\text { neonatal } \\
\text { infection }\end{array}$ \\
\hline 1991 (April-Dec) & 2 & 7,380 & 0.27 & $2(100 \%)$ \\
1992 & 4 & 8,433 & 0.47 & $4(100 \%)$ \\
1993 & 4 & 10,128 & 0.39 & $2(50 \%)$ \\
1994 & 3 & 11,873 & 0.25 & $2(66 \%)$ \\
1995 & 5 & 12,606 & 0.40 & $5(100 \%)$ \\
1996 & 6 & 13,952 & 0.43 & $6(100 \%)$ \\
1997 & 6 & 14,597 & 0.41 & 0 \\
1998 & 5 & 13,475 & 0.37 & $2(40 \%)$ \\
1999 & 5 & 14,051 & 0.36 & 2 \\
$2000(J a n-M a r)$ & 3 & 4,746 & 0.63 & 1 \\
Total & $\mathbf{4 3}$ & $\mathbf{1 1 1 , 2 4 1}$ & $\mathbf{0 . 3 9}$ & $\mathbf{2 6} \quad(\mathbf{6 0 \%})$ \\
\hline
\end{tabular}

Table 2. Demographic data of the 43 cases of sepsis and/or neonatal meningitis by Group B Streptococcus

\begin{tabular}{lrc}
\hline Maternal data & Number of cases (\%) \\
\hline Pre-natal care & 43 & $(100 \%)$ \\
Primipara & 26 & $(60 \%)$ \\
Gestational age & & \\
$\quad 23-34$ weeks & 13 & $(30 \%)$ \\
$\quad 35-36$ weeks & 13 & $(30 \%)$ \\
$\quad 37-40$ weeks & 17 & $(40 \%)$ \\
Antibiotics during labor & 0 & \\
Caesarean Section & 28 & $(65 \%)$ \\
Weight at birth & & \\
$\quad<1,500 \mathrm{~g}$ & 3 & $(7 \%)$ \\
$\quad 1,500-2,499 \mathrm{~g}$ & 15 & $(35 \%)$ \\
$\quad>2,500 \mathrm{~g}$ & 25 & $(58 \%)$ \\
Weight/gestational age & & \\
$\quad$ Adequate & 40 & $(93 \%)$ \\
$\quad$ Small & 1 & $(2 \%)$ \\
Males & 19 & $(44 \%)$ \\
Apgar score & & \\
$\quad<7$ at the $1^{\text {st }}$ minute & 18 & $(42 \%)$ \\
$\quad<7$ at the $5^{\text {th }}$ minute & 5 & $(12 \%)$ \\
\hline
\end{tabular}


Table 3. Maternal Risk factors present in the 43 cases of neonatal infection by Group B Streptococcus

\begin{tabular}{lc}
\hline & Patients (\%) \\
\hline Absent & $14(33 \%)$ \\
One risk factor & 18 \\
GA $<37$ weeks & 1 \\
MR $>18$ hours & 2 \\
Maternal Temperature $>38^{\circ} \mathrm{C}$ & \\
Two risk factors & 6 \\
GA $<37$ weeks and MR $>18 \mathrm{~h}$ & 1 \\
GA $<37$ weeks and Temperature $>38^{\circ} \mathrm{C}$ & 1 \\
Three risk factors & \\
$\quad$ GA $<37$ weeks, MR $>18 h$ and Temp. $>38^{\circ} \mathrm{C}$ & \\
\hline GA $=$ Gestational Age; $\mathrm{MR}=$ membrane rupture. &
\end{tabular}

through maternal anamnesis and clinical exams used to register the development of this invasive neonatal disease.

The incidence we found of 0.39 cases of early GBS neonatal sepsis per 1000 neonates was constant over this last decade of the $20^{\text {th }}$ century. This rate is lower than those observed in developed countries before antibiotic prophylaxis during birth was applied $[1-3,6,7,9,12]$. These countries reported an incidence of 0.7 to 3.7 per 1,000 neonates within the first seven days of life. We analyzed the occurrence of infections only during the first 72 hours after birth. The hospital's infection control staff routinely investigates any clinical suspicion of infection through cultures of all neonates, even those with good clinical indications, during their stay in the hospital. Although the disease may manifest itself between the $4^{\text {th }}$ and $6^{\text {th }}$ day after birth, a great majority of neonates return to the hospital if anything occurs after hospital discharge. In addition, there may be cases of sepsis and/or neonatal meningitis with negative GBS blood culture, depending on the phase of the inflammatory reaction during which the exam was performed. In $30 \%$ to $50 \%$ of the cases of neonatal sepsis, it is not possible to detect the etiological agent. This means that the actual frequency of this disease is probably higher.
The low invasive neonatal infection incidence of 0.39 per 1,000 neonates may be due to the low prevalence of maternal colonization by GBS. In this hospital, GBS was detected in the vaginal swab of $13 \%$ of 406 pregnant women in premature labor from 1995 to 1996 , with no cases of infection of the babies born of these mothers [29].

Though we found a low incidence of invasive neonatal infection, lethality was high; from 1991 to 1996 it reached $100 \%$, decreasing to $40 \%$ from 1997 to 2000. In developed countries, the mortality rate due to this disease declined from $55 \%$ in 1970 to $15 \%$ in the 80 's and $5 \%$ during the last few years [30,31]. The drop in lethality was partially due to more intensive neonatal care, but also may be a result of the institution of antibiotic prophylaxis during labor in women colonized by GBS and/or women presenting one or more risk factors for neonatal GBS infection. Forty two percent of the neonate patients we studied required oxygen therapy at birth, whereas two of them required vasoactive drugs. This fact attests to the poor health of the neonates at birth. Also, all 43 neonates affected by the disease underwent intensive therapy and $77 \%$ of them eventually had septic shock during the first few hours after birth, and a great number died on the second day after birth. This unfavorable clinical evolution might be due to many factors, such as: GBS virulence, 
maternal antibodies, inoculum size and bacterial serotype. Some reports recognize GBS serotype III as the most frequent, accounting for $61 \%$ of the cases in developed countries and associated with serious neonatal infection [3]. The conditions that might justify the elevated lethality of GBS neonatal infection have not yet been investigated in Brazil and constitute an open field for research in perinatology.

A large proportion (26\%) of the 43 patients had meningitis. Thus, whenever a systemic infection is suspected, with an unclear meningitis diagnosis, the cerebrospinal liquor should be investigated. Meningitis requires prolonged antibiotic therapy and has a somber prognosis in terms of its neurological sequels [3,32].

As for the maternal risk factors detected through anamnesis and clinical examination, $60 \%$ of the patients were primipara, $60 \%$ of the neonates were premature and $42 \%$ weighed less than $2500 \mathrm{~g}$ at birth. These data are similar to those reported in American and European studies [5,9,14].

Surprisingly, amniorrhexis longer than 18 hours and maternal temperature during labor higher than $38^{\circ} \mathrm{C}$ were detected in only $16 \%$ and $9 \%$ of the cases, respectively. As for the relationships with previous pregnancies, only two patients reported stillborn babies whereas one reported neonatal death due to an unknown cause.

No risk factors could be assigned to $14 \%$ of the patients. However, although all patients received prenatal medical care, their records did not show any reference to a search for GBS in the urine, vagina and/or rectum during gestation and/or labor. If these exams had been carried out, they would have certainly detected asymptomatic bacteriuria and vaginal and/or rectal colonization by GBS, which would give the opportunity of starting antibiotic prophylaxis during labor.

We conclude that the incidence of early neonatal infection by GBS in Brazil is low when compared to the situation in developed countries, before antibiotic prophylaxis was applied during labor. Despite its low incidence, GBS has been the main agent identified in blood culture whenever the maternity hospital staff suspects neonatal sepsis [13]. Due to the elevated risk of death, it is necessary to prevent invasive disease by GBS. Many studies have shown that this practice significantly reduces GBS vertical transmission and neonatal sepsis. Based on these facts, in 1996, The American Academy of Pediatrics, the American College of Obstetricians and Gynecologists and the Centers for Disease Control and Prevention created guidelines for the perinatal prevention of GBS infection. [11,17,18,30,34,35]. These institutions suggested the use of intravenous antibiotics during labor, using two strategies. The first one recommends a routine search for GBS with anogenital swabs in all women between the $35^{\text {th }}$ and the $37^{\text {th }}$ weeks of pregnancy but do not recommend anti-microbial therapy for the positive cases before the onset of labor. The second strategy, regardless of the results of the anogenital swab, considers several maternal risk factors (premature labor, membrane rupture equal to or longer than 18 hours or maternal temperature equal to or higher than $38^{\circ} \mathrm{C}$ ) as an indication for labor antibiotic therapy. In cases when the patient has had a previous child with invasive GBS infection or bacteriuria by GBS during pregnancy, antibiotic prophylaxis is also indicated during labor $[18,19,28,35,36]$.

Chemoprophylaxis during labor must consist of intravenous penicillin $(5,000,000 \mathrm{U}$ at the beginning and 2,5000,000 U each 4 hours until birth). G penicillin is most widely used because of its narrow spectrum and because of lessened microbial resistance. If the patient is allergic to penicillin, $900 \mathrm{mg}$ of clindamycin may be injected intravenously every 8 hours or $500 \mathrm{~g}$ of erythromycin every 6 hours until birth [1719,21,22,31,35,36].

In the United States the reduction in neonatal sepsis by GBS in the last decade has been attributed to chemo prophylaxis during labor in women who present risk factors and/or are found to have GBS in the urine, vagina and/or rectum (11,13,1619,21,22,28,31,36,37]. Prophylactic antibiotics during labor should have been recommended for 29 (67\%) out of the 43 patients who presented some kind of risk factor, even if GBS cultures were not made from urine, vagina or rectal swabs. 
The conclusion is that, in spite of its low incidence among this Brazilian population, efforts should be made to reduce morbidity and lethality associated with precocious invasive GBS neonatal disease. The determination of risk factors through the patient's anamnesis and clinical exams along with urine, vaginal and rectal cultures for GBS should be stimulated among health care professionals who are in charge of prenatal care. This will permit the identification of women colonized by GBS and the institution of intravenous antibiotic prophylaxis during labor, which would certainly reduce neonatal infection.

\section{References}

1. Brian M.M., Rodney G.B. Group B Streptococcus and pregnancy. Pediatr Ann 1996; 15:206-14.

2. Mckenna D.S., Iams J.D. Group B streptococcal infections. Semin Perinatol 1998;22:267-76.

3. Baker C.J., Edwards M.S. Group B streptococcal infections. In: Remington J., Klein J.O. Infectious Diseases of the Fetus and Newborn Infant. $5^{\text {th }}$ Ed. Philadelphia, Saunders, 2001:1091-156.

4. Boyer K.M., Gotoff S.P. Antimicrobial prophylaxis of neonatal group B streptococcal sepsis. Clin Perinatol 1988; $15: 831-50$.

5. Lim C.T., Thong M.K., Parasakthi N., Ngeow Y.K. Group B Streptococcus: maternal carriage rate and early neonatal septicaemia. Ann Acad Med 1997;26:421-5.

6. Moses L.M., Heath P.T., Wilkinson A.R., et al. Early onset group B streptococcal neonatal infection in Oxford 1985-96. Arch Dis Child Fetal Neonatal Ed 1998;79:F148-9.

7. Eidelman A.I., Rudensky B., Turgeman B., et al. Epidemiology of group B streptococcal colonization and disease in mothers and infants: update of ongoing 10-year Jerusalem study. Isr J Med Sci 1990;26:71-3.

8. Adams W.G., Kinney J.S., Schuchat A. et al. Outbreak of early onset group B streptococcal sepsis. Pediatr Infect Dis J 1993; 12 :565-70.

9. Weisman L.E., Stoll B., Cruess D.F., et al. Early-onset group B streptococcal sepsis: A current assessment. The Journal of Pediatrics 1992;121:428-33.

10. Embleton N., Wariyar U., Hey E. Mortality from early onset group B streptococcal infection in the United Kingdom. Arch Dis Child Fetal Neonatal Ed 1999;80:F139-41.

11. Jeffery H.E. Perinatal group B streptococcal infection: a significant public health problem. Sem Neonatal 1996; $1: 77-89$.
12. Hickman M.E., Rench M.A., Ferrieri P., Baker C. Changing epidemiology of group B streptococcal colonization . Pediatrics 1999; 104:203-9.

13. Baker C.J. Group B streptococcal infections. Clin Perinatol 1997;24:59-70.

14. Schuchat A., Robinson K.D., Plikaytis B.D. et al. Multistate case-control study of maternal risk factors for neonatal Group B streptococcal disease. Pediatr Infect Dis J 1994;13:623-9.

15. Benitz W.E., Gould J.B., Druzin M.L. Risk factors for earlyonset group B streptococcal sepsis: estimation of odds ratios by critical literature review. Pediatrics 1999; 103:77-98.

16. Schimmel M.S., Samueloff A., Eidelman A.I. Prevention of neonatal group B streptococcal infections. is there a rational prevention strategy? Clin Perinatol 1998; $25: 687-97$.

17. Schuchat A., Whitney C., Zangwill K. Prevention of perinatal group B streptococcal disease: a public health perspective. MMWR 1996;45:1-24.

18. Committee on Infectious Diseases and Committee on Fetus and Newborn (American Academy of Pediatrics). Revised guidelines for prevention of early-onset group B streptococcal (GBS) infection. Pediatrics 1997;99:489-96.

19. Committee on Obstetric Practice. ACOG Committee opinion. Prevention of early-onset group B streptococcal disease in newborns. Intern J Gynecol Obstetr 1996;54:197-205.

20. Schuchat A., Zywicki S.S., Dinsmoor M.J., et al. Risk factors and opportunities for prevention of early-onset neonatal sepsis: a multicenter case-control study. Pediatrics 2000; 105;21-6.

21. Benitz W.E., Gould J.B., Druzin M.L. Preventing earlyonset group B streptococcal sepsis: strategy development using decision analysis. Pediatrics 1999;103:76-103.

22. Benitz W.E., Gould J.B., Druzin M.L. Antimicrobial prevention of early-onset group B streptococcal sepsis: estimates of risk reduction based on a critical literature review. Pediatrics 1999;103:78-98.

23. Garner J.S., Jarvis W.R., Emori T.G., et al. CDC definitions for nosocomial infections. Am J Infect Control 1998; $16: 28-40$.

24. Ruoff K.L. Streptococcus. In: Murray P.R. Manual of Clinical Microbiology. $6^{\text {th }}$ Ed. Washington DC, American Society for Microbiology, 1997:299-307.

25. Roselli C.A.M., Segre C.A.M. Classificação do RN. In: Segre C.A.M., Armellini P.A, Marini W.T. RN. $4^{\mathrm{a}}$ Ed. São Paulo, Sarvier 1995:24-34.

26. Andreu A., Barranco M., Bosch J., et al. Prevention of perinatal group B streptococcal disease in Europe. Scand J Infect Dis 1997;29:532. 
27. Schuchat A. Neonatal group B streptococcal diseasescreening and prevention. N Eng J Med 2000;343:209-10.

28. Early-onset group B streptococcal disease - United States, 1998-1999. MMWR 2000;49:793-6.

29. Richtmann R., Kusano E.J., Vaciloto E.L., et al. Colonização pelo estreptococo do grupo B em gestantes: realidade ou conjectura? V Congresso Brasileiro de Controle de Infecção Hospitalar, Rio de Janeiro, 1996.

30. Halsey N.A., Schuchat A., Oh W., Baker C.J. The 1997 AAP guidelines for prevention of early-onset group B streptococcal disease. Pediatrics 1997;100:383-4.

31. Schrag S.J., Zywicki S., Farley M.M., et al. Group B streptococcal disease in the era of intrapartum antibiotic prophylaxis. N Eng J Med 2000;342:15-20.

32. Schuchat A. Group B streptococcus. Lancet 1999;2:51-6.

33. Costa M.L.M., Richtmann R., Takagi N.B., et al. Etiologia de infecção primária da corrente sanguínea em recémnascidos de alto risco. III Congresso Pan-Americano e VII Congresso Brasileiro de Controle de Infecções e Epidemiologia Hospitalar, Belo Horizonte, 2000.

34. Jacobi P., Goldstick O., Sujov P., Eldor J.I. New CDC guidelines for prevention of perinatal group B streptococcal disease. Lancet 1996;348:969.

35. Rose V.L. AAP issues revised guidelines for the prevention of early-onset group B streptococcal infection. Am Fam Phys 1997;1:961-5.

36. Schrag S.J., Whitney C.G., Schuchat A. Neonatal group B streptococcal disease: how infection control teams can contribute to prevention efforts. Infect Control Hosp Epidemiol 2000;21:473-83.

37. Isaacs D., Royle J.A. for the Australian study group for neonatal infections intrapartum antibiotics and early onset neonatal sepsis caused by group B Streptococcus and by other organisms in Australia. Pediatr Infect Dis J 1999; 18:524-8. 